\title{
Fístula gastrobrônquica como complicação rara de gastroplastia para obesidade. Relato de dois casos*
}

\author{
Gastrobronchial fistula as a rare complication of gastroplasty for obesity. \\ A report of two cases
}

\author{
Josemberg Marins Campos ${ }^{1}$, Luciana Teixeira de Siqueira ${ }^{2}$, Marconi Roberto de Lemos Meira $^{3}$, \\ Álvaro Antônio Bandeira Ferraz ${ }^{4}$, Edmundo Machado Ferraz ${ }^{5}$, Murilo José de Barros Guimarães ${ }^{6}$
}

\section{Resumo}

A fístula gastrobrônquica é uma condição rara como complicação decorrente de cirurgia da obesidade. 0 seu manejo exige a participação ativa de um pneumologista, o qual deve conhecer alguns aspectos dos principais tipos de cirurgia bariátrica. Neste relato, descrevemos dois casos de pacientes que apresentaram abscessos subfrênico e pulmonar recidivantes secundários a fístula no ângulo de His durante 19,5 meses, em média. Após o insucesso das relaparotomias, a cura foi obtida por meio da antibioticoterapia e, principalmente, por meio da estenostomia e da dilatação endoscópica, além do uso de clipes e cola de fibrina na fístula. Estas complicações pulmonares não devem ser tratadas isoladamente sem uma avaliação gastrintestinal pois isso pode resultar em piora do quadro respiratório, dificultando o manejo anestesiológico durante procedimentos endoscópicos.

Descritores: Fístula; Abscesso pulmonar; Abscesso subfrênico; Obesidade, cirurgia; Endoscopia.

\begin{abstract}
Gastrobronchial fistula is a rare condition as a complication following bariatric surgery. The management of this condition requires the active participation of a pulmonologist, who should be familiar with aspects of the main types of bariatric surgery. Herein, we report the cases of two patients who presented recurrent subphrenic and lung abscess secondary to fistula at the angle of His for an average of 19.5 months. After relaparotomy was unsuccessful, cure was achieved by antibiotic therapy and, more importantly, by stenostomy and endoscopic dilatation, together with the use of clips and fibrin glue in the fistula. These pulmonary complications should not be treated in isolation without a gastrointestinal evaluation since this can result in worsening of the respiratory condition, thus making anesthetic management difficult during endoscopic procedures.
\end{abstract}

Keywords: Fistula; Lung abscess; Subphrenic abscess; Obesity, surgery; Endoscopy.

\footnotetext{
* Trabalho realizado no Serviço de Cirurgia Geral do Hospital das Clínicas da Universidade Federal de Pernambuco - UFPE - e no Serviço de Pneumologia e Cirurgia Digestiva do Hospital Memorial São José, Recife (PE) Brasil.

1. Doutor em Cirurgia. Universidade Federal de Pernambuco - UFPE - Recife (PE) Brasil.

2. Profa. de Iniciação à Clínica e Técnica Cirúrgica. Universidade Federal de Pernambuco - UFPE - Recife (PE) Brasil.

3. Mestre em Cirurgia. Universidade Federal de Pernambuco - UFPE - Recife (PE) Brasil.

4. Prof. Adjunto de Cirurgia. Universidade Federal de Pernambuco - UFPE - Recife (PE) Brasil.

5. Prof. Titular de Cirurgia Abdominal e Bases da Técnica Cirúrgica. Universidade Federal de Pernambuco - UFPE - Recife (PE) Brasil.

6. Prof. de Pneumologia da Faculdade de Ciências Médicas. Universidade Federral de Pernambuco - UFPE - Recife (PE) Brasil.

Endereço para correspondência: Josemberg Marins Campos. Rua Joaquim Nabuco, 92, 1101, Graças, CEP 51011-000, Recife, PE, Brasil.

Tel/Fax 5581 3441-4460. E-mail: berg@elogica.com.br

Recebido para publicação em 20/3/06. Aprovado, após revisão, em 8/8/06.
} 


\section{Introdução}

A obesidade é uma doença crônica associada a comorbidades a qual deve ser controlada por meio de exercício físico e mudança de hábitos alimentares, sendo indicado tratamento cirúrgico quando as medidas clínicas forem ineficientes. ${ }^{(1)}$

Existem várias técnicas operatórias para controle da obesidade, mas a gastroplastia a Fobi-Capella tem sido a mais empregada. Recentemente, também vem sendo executada uma outra técnica, conhecida como sleeve gastrectomy em inglês, que envolve apenas a ressecção parcial e vertical do estômago, o qual é transformado num tubo para reduzir o reservatório do órgão.

Embora as duas técnicas cirúrgicas apresentem bom resultados em relação à perda de peso e ao controle de comorbidades, elas podem evoluir com complicações pós-operatórias precoces, sendo a fístula gástrica uma das mais graves devido à ocorrência de sepse abdominal e de alterações respiratórias, principalmente no pulmão esquerdo, decorrentes da freqüente existência de abscesso subfrênico. ${ }^{(2)}$ Estas conseqüências sobre o aparelho respiratório exigem a participação efetiva de um especialista em pneumologia, o qual deve conhecer alguns aspectos dos principais tipos de cirurgia bariátrica, incluindo as complicações operatórias.

Habitualmente, o processo infeccioso subfrênico provoca fístula gastrocutânea, derrame pleural e infecção respiratória. ${ }^{(3)}$ Porém, na literatura, há carência de relatos de ocorrência de fístula gastrobrônquica após cirurgia da obesidade. Assim, objetiva-se apresentar a evolução satisfatória de dois pacientes com abscesso pulmonar decorrente de fístula do ângulo de His após gastroplastia.

\section{Relato dos casos}

\section{Caso 1}

Um paciente do sexo masculino com 44 anos de idade submeteu-se à cirurgia de Fobi-Capella por videolaparoscopia para controle da obesidade mórbida, sendo reoperado após $48 \mathrm{~h}$ para tratamento de obstrução intestinal. Dois meses depois, foram realizadas duas sessões de dilatação endoscópica com balão para correção de estenose da anastomose gastrojejunal. Em seguida, o paciente apresentou quadro de vômica, tosse produtiva, febre e dor à inspiração profunda na região tóraco-abdominal esquerda, sendo diagnosticada uma imagem nodular de $7 \mathrm{~cm}$ de diâmetro, em base pulmonar esquerda, por meio de tomografia axial computadorizada de tórax (Figura 1a). 0 paciente apresentou melhora clínica após antibioticoterapia, fisioterapia respiratória e acompanhamento no serviço de pneumologia. Porém, houve duas recidivas num intervalo de três meses, quando então foi diagnosticada uma fístula gastrobrônquica por meio de imagem radiológica (Figura 1b) e endoscópica (Figura 1c) que evidenciou um orifício com $10 \mathrm{~mm}$ de diâmetro no ângulo de His, através do qual foi possível a passagem do endoscópio até uma pequena cavidade subfrênica onde se visualizava um orifício interno do trajeto fistuloso para a árvore brônquica. Não houve sucesso na tentativa de correção via laparotomia, sendo realizada apenas uma gastrostomia no estômago excluso para nutrição. Assim, usando bisturi elétrico, realizou-se a secção endoscópica da estenose gástrica e do septo situado entre a fístula e a bolsa gástrica associada à dilatação com balão de 20 e $30 \mathrm{~mm}$ (Boston Microvasive, Boston, MA, EUA) e aplicação de clipes na fístula (Olympus Corp., Tokyo, Japão). Após seis meses, observou-se aumento do diâmetro da bolsa gástrica, desaparecimento do referido septo e fechamento definitivo da fístula com resolução do abscesso pulmonar.

\section{Caso 2}

Uma paciente com 44 anos de idade foi submetida à gastroplastia vertical com anel (sleeve gastrectomy) por videolaparoscopia, evoluindo com fístula gástrica e cinco episódios de abscesso subfrênico durante 8 meses. Seu quadro clínico se iniciava com dor no ombro esquerdo e evoluía com febre, tosse, leucocitose, queda do estado geral e perda de peso excessiva. Os dois últimos episódios foram acompanhados de tosse com expectoração purulenta e a tomografia axial computadorizada de tórax diagnosticou abscesso na base pulmonar esquerda (Figura 2a), secundário a abscesso subfrênico a partir do ângulo de His, e estenose gástrica ao nível do anel e da incisura angularis. 0 abscesso pulmonar proveniente da fístula gastrobrônquica foi drenado através de cateter visualizado em radiografia contrastada (Figura 2b) e a imagem endoscópica mostra o orifício interno da fístula no ângulo de His (Figura 2c). 

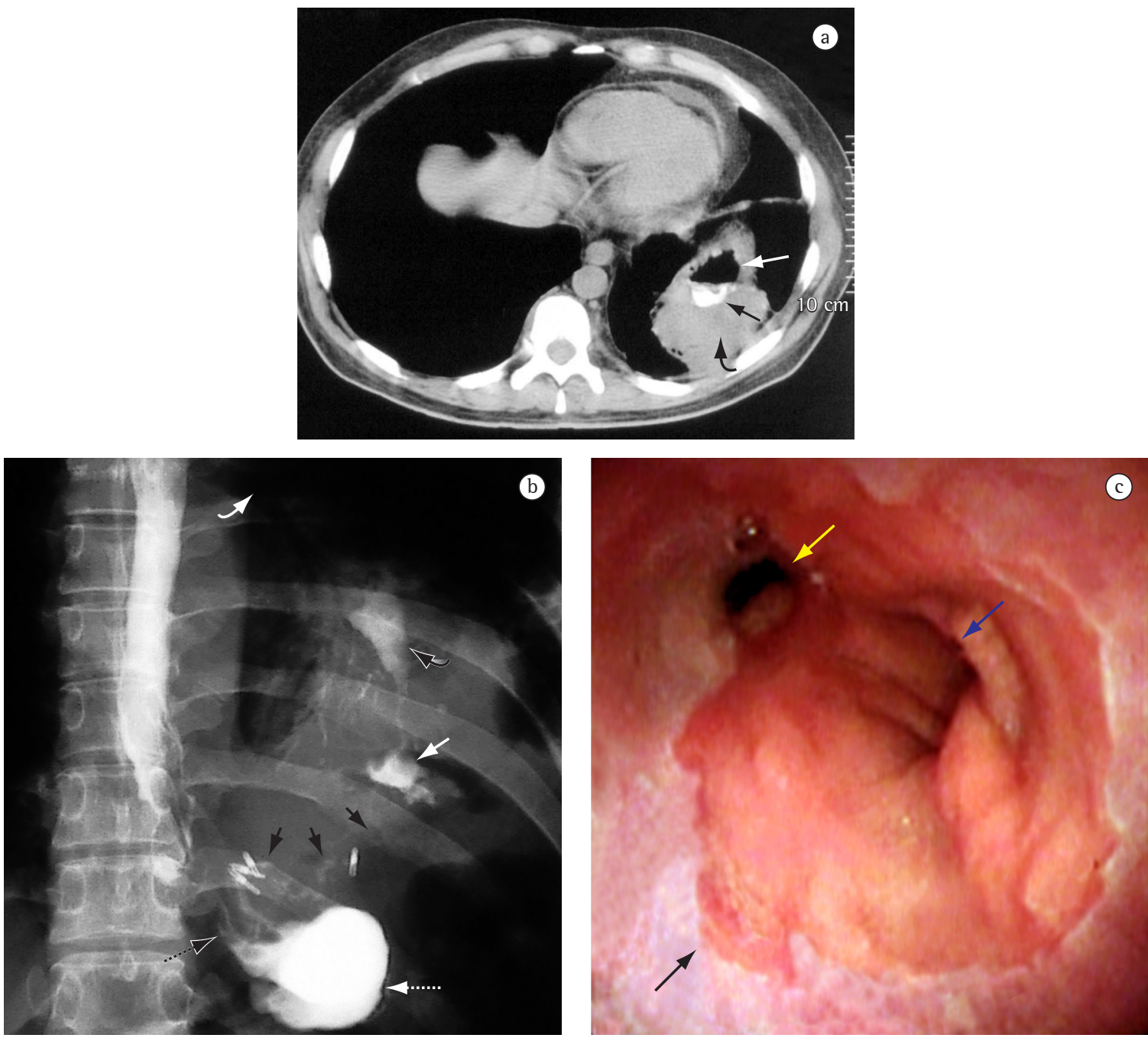

Figura 1 - Caso 1: a) Tomografia axial computadorizada de tórax: abscesso na base do pulmão esquerdo (seta preta curva) contendo ar (seta branca), e contraste proveniente da bolsa gástrica (seta preta); b) Raio-x contrastado: fístula a partir do ângulo de His com clipes (setas pretas), bolsa gástrica (seta preta tracejada), alça jejunal (seta branca tracejada), abscesso subfrênico (seta branca), abscesso pulmonar (seta preta curva), e árvore brônquica (seta branca curva); e c) Imagem endoscópica: transição esofagogástrica (seta preta), bolsa gástrica (seta azul), e fístula no ângulo de His (seta amarela).

A paciente recebeu acompanhamento especializado de um pneumologista, um endoscopista e um cirurgião, sendo tratada por meio de antibioticoterapia, fisioterapia respiratória e suporte nutricional por sonda nasoenteral. Além disso, foi submetida a uma laparoscopia exploradora para retirada do anel, quando foi evidenciado um intenso bloqueio inflamatório na região subfrênica esquerda e na transição esofagogástrica o qual impossibilitava a abordagem cirúrgica da fístula.
Assim, foram realizadas seis sessões endoscópicas de estenostomia com bisturi elétrico e dilatação da área estenótica, o que permitiu a ingestão satisfatória de alimentos, o restabelecimento nutricional e a cicatrização do abscesso pulmonar, após o fechamento definitivo da fístula.

\section{Discussão}

A fístula gástrica após cirurgia de obesidade ocorre em 0,9 a 2,6\% dos casos, podendo chegar 

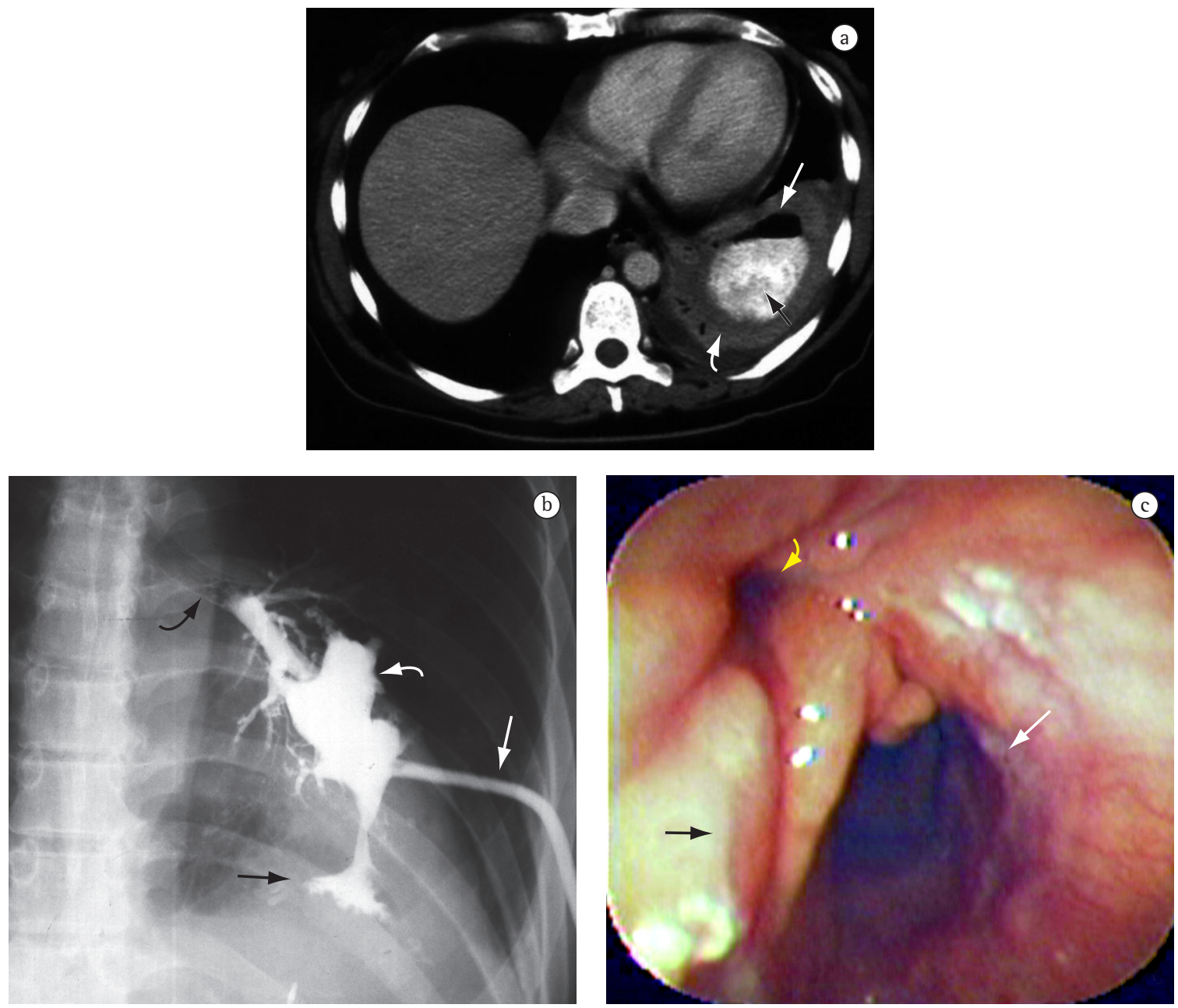

Figura 2 - Caso 2: a) Tomografia axial computadorizada de tórax: abscesso na base do pulmão esquerdo (seta branca curva) contendo ar (seta branca) e contraste proveniente da bolsa gástrica (seta preta); b) Raio-x contrastado: cateter (seta branca) drenando abscesso pulmonar (seta branca curva), abscesso subfrênico (seta preta), e árvore brônquica (seta preta curva); e c) Imagem endoscópica: transição esofagogástrica (seta preta); bolsa gástrica (seta branca); e fístula no ângulo de His (seta amarela).

a 8\% nas reoperações, e localiza-se com maior freqüência ao nível do ângulo de His, provavelmente em decorrência de isquemia no ápice do grampeamento lateral. ${ }^{(1)}$

A secreção da fístula segue para a região subfrênica e pode causar sepse abdominal, complicações no pulmão esquerdo e fístula gastrocutânea. Menos comumente, ocorre drenagem para o estômago excluso (fístula gastrogástrica), ${ }^{(3)}$ enquanto que a drenagem para a arvore brônquica (fístula gastrobrônquica) tem sido referida na literatura como complicação de trauma, cirurgia esofago- gástrica (esofagectomia transhiatal por neoplasias ou megaesôfago), esplenectomia e úlcera gástrica perfurada. ${ }^{(4,5)}$ Nessas cirurgias abdominais, há desenvolvimento de abscesso subfrênico que ultrapassa o diafragma por via linfática, causando derrame pleural, fístula gastrobrônquica e abscesso pulmonar à esquerda. ${ }^{(4)}$ Seguramente, isto também ocorreu em nosso estudo.

Os pacientes em análise apresentaram aspectos em comum que facilitaram a comunicação da secreção do estômago com o sistema respiratório. Houve estenose da bolsa gástrica ou da anastomose 
gastrojejunal a qual aumentava a pressão e direcionava os alimentos para a fístula no ângulo de His, levando ao surgimento de infecção subfrênica recidivante, na ausência de adequada via de saída para a pele, uma vez que o dreno abdominal já havia sido retirado.

Esses dois pacientes fazem parte de um grupo de 27 pacientes portadores de fístula gástrica tratados por via endoscópica. A característica específica dos dois casos era o caráter recidivante da fístula por tempo prolongado, a estenose da bolsa gástrica ou da anastomose gastrojejunal e a ausência de uma via de drenagem da infecção abdominal.

Em associação à endoscopia digestiva e aos exames de imagem de tórax e abdome, a broncoscopia tem sido apresentada como método diagnóstico e terapêutico em casos de fístula gastrobrônquica, principalmente após esofagectomia, o que facilita a avaliação e a limpeza adequada das vias aéreas. ${ }^{(4,5)}$ No estudo atual, não houve necessidade deste procedimento, considerando-se a rápida melhora do quadro respiratório após a antibioticoterapia, a suspensão da dieta oral e o início da dieta por gastrostomia ou sonda nasoenteral.

0 tratamento clássico de fístula digestiva inclui suporte nutricional e ressecção cirúrgica do trajeto fistuloso, a qual se associa a considerável morbidade devido ao intenso processo inflamatório no abdome superior, além do risco de lesão das estruturas adjacentes, tornando-se um procedimento laborioso em decorrência das freqüentes reoperações, ${ }^{(1)}$ o que leva a infecção de ferida e suas conseqüências. Assim, o tratamento endoscópico se apresenta como um meio minimamente invasivo e eficaz para a resolução da estenose gástrica, que é considerada a principal causa de perpetuação da fístula, como demonstrado neste estudo.

Além dos métodos terapêuticos endoscópicos utilizados no referido trabalho, o uso da prótese esofágica ou traqueobrônquica, a qual é aplicada em fistula gastrobrônquica pós-esofagectomia, tem sido implementado. ${ }^{(6)}$ Nos dois pacientes em análise, o uso da prótese plástica auto-expansível não foi indicado em decorrência da exígua literatura existente sobre o assunto naquele momento. Recentemente, utilizamos este método com sucesso em dois pacientes portadores de fístula gástrica com diâmetro maior do que $10 \mathrm{~mm}$ pois ele tem a vantagem de diminuir o número de sessões endoscópicas, além de permitir a ingestão precoce de alimentos, o que está de acordo com a literatura atual..$^{(7)}$

Considerando-se a complexidade do paciente obeso e do processo infeccioso gastrobrônquico, é fundamental a avaliação conjunta de especialistas, incluindo pneumologistas, cirurgiões, radiologistas e endoscopistas.

A abordagem específica das complicações pulmonares sem o estudo da estenose e da fístula gástrica retarda a resolução da infecção subfrênica e resulta em piora do quadro respiratório, dificultando o manejo anestesiológico durante a execução de procedimentos cirúrgicos e endoscópicos que visam à cura da fístula e dos problemas respiratórios.

Assim, diante de infecção respiratória persistente em pós-operatório precoce ou tardio de cirurgia da obesidade, deve-se proceder sem demora à avaliação gastrintestinal por meio de exames de imagem e por via endoscópica visando o diagnóstico e o planejamento terapêutico de uma possível fístula a partir do estômago operado.

\section{Referências}

1. Rocha LCM, Lima Jr. GF. Papel da endoscopia na obesidade mórbida. In: Savasi-Rocha PR, Coelho LGV, Diniz MTC, Nunes TA, editores. Tópicos em Gastroenterologia - 13. Rio de Janeiro: Editora Medsi; 2003. p.53-75.

2. Merkle EM, Hallowell PT, Crouse C, Nakamoto DA, Stellato TA. Roux-en-Y gastric bypass for clinically severe obesity: normal appearance and spectrum of complications at imaging. Radiology. 2005;234(3):674-83.

3. Stanczyk M, Deveney CW, Traxler SA, McConnell DB, Jobe BA, O'Rourke RW. Gastro-gastric fistula in the era of divided Roux-en-Y gastric bypass: strategies for prevention, diagnosis and management. Obes Surg. 2006;16(3):359-64.

4. Jeganathan R, Pore N, Clements WD. Gastrobronchial fistula - a complication of splenectomy. Ulster Med J. 2004;73(2):126-8.

5. Purucker EA, Sudfeld S, Matern S. Gastrobronchial fistula after caustic injury due to lye ingestion. Endoscopy. 2003;35(3):252.

6. Bennie MJ, Sabharwal T, Dussek J, Adam A. Bronchogastric fistula successfully treated with the insertion of a covered bronchial stent. Eur Radiol. 2003;13(9):2222-25.

7. Maluf-Filho F, Moura EGH. Prótese plástica auto-expansível no tratamento da fístula complexa pós-cirurgia bariátrica. GED. 2005;24(4):203. 